\title{
Algebras of symmetric analytic functions on Cartesian powers of Lebesgue integrable in a power $p \in[1,+\infty)$ functions
}

\begin{abstract}
Vasylyshyn T.V.
The work is devoted to the study of Fréchet algebras of symmetric (invariant under the composition of every of components of its argument with any measure preserving bijection of the domain of components of the argument) analytic functions on Cartesian powers of complex Banach spaces of Lebesgue integrable in a power $p \in[1,+\infty)$ complex-valued functions on the segment $[0,1]$ and on the semi-axis. We show that the Fréchet algebra of all symmetric analytic entire complex-valued functions of bounded type on the $n$th Cartesian power of the complex Banach space $L_{p}[0,1]$ of all Lebesgue integrable in a power $p \in[1,+\infty)$ complex-valued functions on the segment $[0,1]$ is isomorphic to the Fréchet algebra of all analytic entire functions on $\mathbb{C}^{m}$, where $m$ is the cardinality of the algebraic basis of the algebra of all symmetric continuous complex-valued polynomials on this Cartesian power. The analogical result for the Fréchet algebra of all symmetric analytic entire complex-valued functions of bounded type on the $n$th Cartesian power of the complex Banach space $L_{p}[0,+\infty)$ of all Lebesgue integrable in a power $p \in[1,+\infty)$ complex-valued functions on the semi-axis $[0,+\infty)$ is proved.

Key words and phrases: symmetric polynomial, symmetric analytic function, Fréchet algebra of analytic functions.
\end{abstract}

Vasyl Stefanyk Precarpathian National University, 57 Shevchenka str., 76018, Ivano-Frankivsk, Ukraine

E-mail: taras.v.vasylyshynegmail.com

\section{Introduction}

Symmetric polynomials and symmetric analytic functions on Banach spaces with symmetric structures were studied by a number of authors [1-10,12-19]. In some cases, an algebra of symmetric continuous polynomials has a countable or even a finite algebraic basis (a subset of an algebra is called an algebraic basis of this algebra if every element of the algebra can be uniquely represented as a linear combination of products of powers of elements of this subset). Since every element of the spectrum (the set of all continuous multiplicative linear functionals) of a topological algebra of analytic functions is uniquely determined by its values on elements of an algebraic basis of a dense subalgebra of polynomials, the knowledge of algebraic bases of algebras of symmetric polynomials is important for the description of spectra of respective topological algebras of symmetric analytic functions (see $[1,3,5,7,12,18]$ ). In turn, in some cases, a topological algebra of analytic functions can be represented as a topological algebra of analytic functions on its spectrum with a proper topologisation (see, e.g., [7]).

In this work we consider symmetric continuous polynomials and symmetric analytic func-

$\mathrm{y} \Delta \mathrm{K} 517.98$

2020 Mathematics Subject Classification:46G20, 46J20.

This research was funded by the National Research Foundation of Ukraine, 2020.02/0025, 0120U103996. 
tions on Cartesian powers of complex Banach spaces $L_{p}[0,1]$ and $L_{p}[0,+\infty)$ of all complexvalued Lebesgue integrable in a power $p$ functions on $[0,1]$ and $[0,+\infty)$ respectively, where $1 \leq p<+\infty$. We represent Fréchet algebras of symmetric entire analytic functions of bounded type on these Cartesian powers as Fréchet algebras of entire analytic functions on their spectra.

\section{Preliminaries}

We denote by $\mathbb{N}$ the set of all positive integers and by $\mathbb{Z}_{+}$the set of all nonnegative integers.

Polynomials. A mapping $P: X \rightarrow \mathbb{C}$, where $X$ is a complex Banach space, is called an $N$-homogeneous polynomial, where $N \in \mathbb{N}$, if there exists an $N$-linear mapping $A_{P}: X^{N} \rightarrow \mathbb{C}$ such that

$$
P(x)=A_{P}(\underbrace{x, \ldots, x}_{N})
$$

for every $x \in X$.

It is known that an $N$-homogeneous polynomial $P: X \rightarrow \mathbb{C}$ is continuous if and only if $\|P\|<+\infty$, where

$$
\|P\|=\sup _{\|x\| \leq 1}|P(x)| .
$$

Consequently, for a continuous $N$-homogeneous polynomial $P: X \rightarrow \mathbb{C}$, we have

$$
|P(x)| \leq\|P\|\|x\|^{N}
$$

for every $x \in X$.

A mapping $P: X \rightarrow \mathbb{C}$ is called a polynomial of a degree at most $N$ if it can be represented in the form

$$
P=P_{0}+P_{1}+\ldots+P_{N}
$$

where $P_{0} \in \mathbb{C}$ and $P_{j}: X \rightarrow \mathbb{C}$ is a $j$-homogeneous polynomial for every $j \in\{1, \ldots, N\}$.

For details on polynomials on Banach spaces, we refer the reader to [11].

Algebraic combinations. Let $T$ be an arbitrary nonempty set. A mapping $f: T \rightarrow \mathbb{C}$ is called an algebraic combination of mappings $f_{1}, \ldots, f_{k}: T \rightarrow \mathbb{C}$ if there exists a polynomial $Q: \mathbb{C}^{k} \rightarrow \mathbb{C}$ such that

$$
f(x)=Q\left(f_{1}(x), \ldots, f_{k}(x)\right)
$$

for every $x \in T$. Mappings $f_{1}, \ldots, f_{k}: T \rightarrow \mathbb{C}$ are called algebraically independent when $Q\left(f_{1}(x), \ldots, f_{k}(x)\right)=0$ for every $x \in T$ if and only if the polynomial $Q$ is identically equal to zero. If mappings $f_{1}, \ldots, f_{k}$ are algebraically independent and polynomials $Q_{1}, Q_{2}: \mathbb{C}^{k} \rightarrow \mathbb{C}$ are such that

$$
Q_{1}\left(f_{1}(x), \ldots, f_{k}(x)\right)=Q_{2}\left(f_{1}(x), \ldots, f_{k}(x)\right)
$$

for every $x \in T$, then the polynomial $Q_{1}$ is identically equal to the polynomial $Q_{2}$. Thus, every algebraic combination of algebraically independent mappings is unique.

A subset $\mathcal{B}$ of some algebra of mappings $\mathcal{A}$ is called an algebraic basis of $\mathcal{A}$ if every element of $\mathcal{A}$ can be uniquely represented as an algebraic combination of some elements of $\mathcal{B}$. Evidently, every algebraic basis is algebraically independent. 
Symmetric functions. Let $p \in[1,+\infty)$ and $n \in \mathbb{N}$. Let $\Omega$ be the Lebesgue measurable subset of $\mathbb{R}$ with positive measure. Let $L_{p}(\Omega)$ be the complex Banach space of (classes of) functions $y: \Omega \rightarrow \mathbb{C}$, for which the $p$ th power of the absolute value is Lebesgue integrable with the norm

$$
\|y\|_{p, \Omega}=\left(\int_{\Omega}|y(t)|^{p} d t\right)^{1 / p} .
$$

Let $\left(L_{p}(\Omega)\right)^{n}$ be the $n$th Cartesian power of $L_{p}(\Omega)$ with the norm

$$
\|y\|_{p, n, \Omega}=\left(\sum_{s=1}^{n} \int_{\Omega}\left|y_{s}(t)\right|^{p} d t\right)^{1 / p},
$$

where $y=\left(y_{1}, \ldots, y_{n}\right) \in\left(L_{p}(\Omega)\right)^{n}$.

Let $\Xi_{\Omega}$ be the set of all bijections $\sigma: \Omega \rightarrow \Omega$ such that both $\sigma$ and $\sigma^{-1}$ are measurable and preserve the Lebesgue measure. A function $f:\left(L_{p}(\Omega)\right)^{n} \rightarrow \mathbb{C}$ is called symmetric if

$$
f\left(\left(y_{1} \circ \sigma, \ldots, y_{n} \circ \sigma\right)\right)=f\left(\left(y_{1}, \ldots, y_{n}\right)\right)
$$

for every $\left(y_{1}, \ldots, y_{n}\right) \in\left(L_{p}(\Omega)\right)^{n}$ and for every $\sigma \in \Xi_{\Omega}$.

Denote $L_{p}[0,1]:=L_{p}([0,1])$ and $L_{p}[0,+\infty):=L_{p}([0,+\infty))$. For every multi-index $k=\left(k_{1}, \ldots, k_{n}\right) \in \mathbb{Z}_{+}^{n}$ such that $1 \leq|k| \leq p$, where $|k|=k_{1}+\cdots+k_{n}$, let us define a mapping $R_{k,[0,1]}:\left(L_{p}[0,1]\right)^{n} \rightarrow \mathbb{C}$ by

$$
R_{k,[0,1]}(y)=\int_{[0,1]} \prod_{\substack{s=1 \\ k_{s}>0}}^{n}\left(y_{s}(t)\right)^{k_{s}} d t,
$$

where $y=\left(y_{1}, \ldots, y_{n}\right) \in\left(L_{p}[0,1]\right)^{n}$. If $p \in \mathbb{N}$, for every multi-index $k=\left(k_{1}, \ldots, k_{n}\right) \in \mathbb{Z}_{+}^{n}$ such that $|k|=p$, let us define a mapping $R_{k,[0,+\infty)}:\left(L_{p}[0,+\infty)\right)^{n} \rightarrow \mathbb{C}$ by

$$
R_{k,[0,+\infty)}(y)=\int_{[0,+\infty)} \prod_{\substack{s=1 \\ k_{s}>0}}^{n}\left(y_{s}(t)\right)^{k_{s}} d t
$$

where $y=\left(y_{1}, \ldots, y_{n}\right) \in\left(L_{p}[0,+\infty)\right)^{n}$.

Note that $R_{k,[0,1]}$ and $R_{k,[0,+\infty)}$ are symmetric continuous $|k|$-homogeneous polynomials.

Theorem 1 ([14, Theorem 2.10]). Let $N \in \mathbb{N}$. Every $N$-homogeneous symmetric continuous polynomial $P:\left(L_{p}[0,1]\right)^{n} \rightarrow \mathbb{C}$ can be uniquely represented as an algebraic combination of polynomials $R_{k,[0,1]}$, where multi-indexes $k \in \mathbb{Z}_{+}^{n}$ are such that $1 \leq|k| \leq \min \{p, N\}$.

Theorem 2 ([15, Theorem 3]). Let $N \in \mathbb{N}$. Let $P:\left(L_{p}[0,+\infty)\right)^{n} \rightarrow \mathbb{C}$ be a symmetric continuous $N$-homogeneous polynomial. If $p \notin \mathbb{N}$ or $N<p$, then $P \equiv 0$. If $p \in \mathbb{N}$ and $N \geq p$, then $P$ can be uniquely represented as an algebraic combination of polynomials $R_{k,[0,+\infty)}$, where $k \in \mathbb{Z}_{+}^{n}$ are such that $|k|=p$.

Theorems 1 implies the following corollary.

Corollary 1. The set of polynomials

$$
\left\{R_{k,[0,1]}: k \in \mathbb{Z}_{+}^{n} \text { such that } 1 \leq|k| \leq p\right\}
$$

is algebraically independent.

Analogically, Theorem 2 implies the following corollary.

Corollary 2. Let $p \in \mathbb{N}$. The set of polynomials

$$
\left\{R_{k,[0,+\infty)}: k \in \mathbb{Z}_{+}^{n} \text { such that }|k|=p\right\}
$$

is algebraically independent. 
Algebras of analytic functions. The set of all continuous multiplicative linear nontrivial functionals (characters) of a topological algebra is called the spectrum of this algebra. Let us denote $\mathcal{M}(\mathcal{A})$ the spectrum of a topological algebra $\mathcal{A}$.

Let $X$ be a complex Banach space. Let $H_{b}(X)$ be the Fréchet algebra of all entire analytic functions $f: X \rightarrow \mathbb{C}$, which are bounded on bounded sets, endowed with the topology of uniform convergence on bounded sets.

For $r>0$ and $f \in H_{b}(X)$ let

$$
\|f\|_{r}=\sup _{\|x\| \leq r}|f(x)| .
$$

Note that the topology of $H_{b}(X)$ is generated by any set of norms $\left\{\|\cdot\|_{r}: r \in U\right\}$, where $U$ is an arbitrary unbounded subset of $(0,+\infty)$.

Note that for every $x \in X$ the functional $\delta_{x}: f \in H_{b}(X) \mapsto f(x) \in \mathbb{C}$, which is called a point-evaluation functional at the point $x$, belongs to the spectrum of the algebra $H_{b}(X)$.

Let $H_{b s}\left(\left(L_{p}(\Omega)\right)^{n}\right)$ be the subalgebra of the Fréchet algebra $H_{b}\left(\left(L_{p}(\Omega)\right)^{n}\right)$, which consists of all symmetric elements of $H_{b}\left(\left(L_{p}(\Omega)\right)^{n}\right)$.

The space $c_{00}^{(m)}\left(\mathbb{C}^{n}\right)$. Let $M$ be a finite nonempty subset of $\mathbb{Z}_{+}^{n}$. Let $\mathbb{C}^{M}$ be the vector space of all mappings from $M$ to $\mathbb{C}$. Note that every element $\xi \in \mathbb{C}^{M}$ can be considered as an $|M|$-dimensional complex vector $\left(\xi_{k}\right)_{k \in M}$, where $\xi_{k}=\xi(k)$ for $k \in M$ and $|M|$ is the cardinality of $M$. Therefore, $\mathbb{C}^{M}$ is isomorphic to $\mathbb{C}^{|M|}$. We endow the space $\mathbb{C}^{M}$ with the norm

$$
\|\xi\|_{\infty}=\max _{k \in M}\left|\xi_{k}\right| .
$$

For $m \in \mathbb{N}$, let $c_{00}^{(m)}\left(\mathbb{C}^{n}\right)$ be the space of all sequences $x=\left(x_{1}, \ldots, x_{m}, 0, \ldots\right)$, where $x_{j}=$ $\left(x_{j}^{(1)}, \ldots, x_{j}^{(n)}\right) \in \mathbb{C}^{n}$ for $j \in\{1, \ldots, m\}$, and $0=(0, \ldots, 0) \in \mathbb{C}^{n}$. We endow the space $c_{00}^{(m)}\left(\mathbb{C}^{n}\right)$ with the norm

$$
\|x\|_{\ell_{p}}=\left(\sum_{j=1}^{m} \sum_{s=1}^{n}\left|x_{j}^{(s)}\right|^{p}\right)^{1 / p} .
$$

For every $k \in \mathbb{Z}_{+}^{n} \backslash\{(0, \ldots, 0)\}$, let $H_{k}^{(m)}: c_{00}^{(m)}\left(\mathbb{C}^{n}\right) \rightarrow \mathbb{C}$ be defined by

$$
H_{k}^{(m)}(x)=\sum_{j=1}^{m} \prod_{\substack{s=1 \\ k_{s}>0}}^{n}\left(x_{j}^{(s)}\right)^{k_{s}} .
$$

For an arbitrary nonempty finite set $M \subset \mathbb{Z}_{+}^{n}$ such that $|k| \geq 1$ for every $k \in M$, let us define a mapping $\pi_{M}^{(m)}: c_{00}^{(m)}\left(\mathbb{C}^{n}\right) \rightarrow \mathbb{C}^{M}$ by

$$
\pi_{M}^{(m)}(x)=\left(H_{k}^{(m)}(x)\right)_{k \in M} \text { for every } \quad x \in c_{00}^{(m)}\left(\mathbb{C}^{n}\right) .
$$

We will use the following result, proved in [9].

Theorem 3 ([9, Theorem 6]). Let $M$ be a finite nonempty subset of $\mathbb{Z}_{+}^{n}$ such that $|k| \geq 1$ for every $k \in M$. Then

(i) there exists $m \in \mathbb{N}$ such that for every $\xi=\left(\xi_{k}\right)_{k \in M} \in \mathbb{C}^{M}$ there exists $x_{\xi} \in c_{00}^{(m)}\left(\mathbb{C}^{n}\right)$ such that $\pi_{M}^{(m)}\left(x_{\xi}\right)=\xi$;

(ii) there exists a constant $\rho>0$ such that if $\|\xi\|_{\infty}<1$, then $\left\|x_{\xi}\right\|_{\ell_{p}}<\rho$ for every $p \in$ $[1,+\infty)$ 
For $m \in \mathbb{N}$, let $J_{m}: c_{00}^{(m)}\left(\mathbb{C}^{n}\right) \rightarrow\left(L_{p}[0,1]\right)^{n}$ be defined by

$$
J_{m}(x)=\left(\sum_{j=1}^{m} x_{j}^{(1)} 1_{\left[\frac{j-1}{m}, \frac{j}{m}\right]}, \ldots, \sum_{j=1}^{m} x_{j}^{(n)} 1_{\left[\frac{j-1}{m}, \frac{j}{m}\right]}\right)
$$

for $x=\left(x_{1}, \ldots, x_{m}, 0, \ldots\right) \in c_{00}^{(m)}\left(\mathbb{C}^{n}\right)$. Note that $J_{m}$ is a linear operator.

Lemma 1 ([14, Lemma 2.6]). For every $x \in c_{00}^{(m)}\left(\mathbb{C}^{n}\right)$,

$$
\left\|J_{m}(x)\right\|_{p, n,[0,1]}=\frac{1}{m^{1 / p}}\|x\|_{\ell_{p}} .
$$

Consequently, $J_{m}$ is continuous. The following lemma is a partial result of [14, Lemma 2.7].

Lemma 2. For every $k \in \mathbb{Z}_{+}^{n}$ such that $1 \leq|k| \leq p$ and $l \in \mathbb{N}$,

$$
R_{k,[0,1]}\left(J_{2^{l}}(x)\right)=\frac{1}{2^{l}} H_{k}^{\left(2^{l}\right)}(x) \quad \text { for every } \quad x \in c_{00}^{\left(2^{l}\right)}\left(\mathbb{C}^{n}\right) .
$$

\section{Symmetric analytic functions on Cartesian powers of $L_{p}[0,1]$ and $L_{p}[0,+\infty)$}

Theorem 4. Let $X$ be a complex Banach space. Let $m \in \mathbb{N}$ and $\Gamma=\left\{P_{1}, P_{2}, \ldots, P_{m}\right\}$ be a set of polynomials on the space $X$, which has the following properties:

1) for every $j \in\{1, \ldots, m\}$ the mapping $P_{j}: X \rightarrow \mathbb{C}$ is a continuous $d_{j}$-homogeneous polynomial, where $d_{j} \in \mathbb{N}$;

2) the set of polynomials $\Gamma$ is algebraically independent;

3) there exists a constant $C>0$ such that for every vector $z=\left(z_{1}, \ldots, z_{m}\right) \in \mathbb{C}^{m}$ there exists an element $x_{z} \in X$ such that $\left\|x_{z}\right\| \leq C\|z\|_{\infty}$ and $P_{j}\left(x_{z}\right)=z_{j}$ for every $j \in\{1, \ldots, m\}$, where $\|z\|_{\infty}=\max \left\{\left|z_{1}\right|, \ldots,\left|z_{m}\right|\right\}$.

Let $\mathcal{A}(X)$ be a closed subalgebra of the Fréchet algebra $H_{b}(X)$ such that for every function $f \in \mathcal{A}(X)$ each term of the Taylor series of this function is an algebraic combination of elements of the set $\Gamma$. Then Fréchet algebras $\mathcal{A}(X)$ and $H\left(\mathbb{C}^{m}\right)$ are isomorphic. The spectrum of the Fréchet algebra $\mathcal{A}(X)$ coincides with the set of all point-evaluation functionals at points of the space $X$.

Proof. Let $f \in \mathcal{A}(X)$. Since $\mathcal{A}(X) \subset H_{b}(X)$, it follows that $f$ is an entire analytic function of bounded type on the space $X$. Therefore there exists the Taylor series $\sum_{n=0}^{\infty} f_{n}$, where $f_{n}$ is a continuous $n$-homogeneous polynomial for every $n \in \mathbb{Z}_{+}$, which is uniformly convergent to the function $f$ on every bounded subset of $X$. By the conditions of the theorem, every polynomial $f_{n}$ can be represented as an algebraic combination of elements of the set $\Gamma$. By the condition 2), such an algebraic combination is unique. Since the polynomial $f_{n}$ is $n$-homogeneous, it follows that this algebraic combination is a linear combination of $n$-homogeneous polynomials of the form $P_{1}^{k_{1}} P_{2}^{k_{2}} \cdots P_{m}^{k_{m}}$, where $k_{1}, k_{2}, \ldots, k_{m} \in \mathbb{Z}_{+}$, (here $\left.0^{0}=1\right)$. Note that the polynomial 
$P_{1}^{k_{1}} P_{2}^{k_{2}} \ldots P_{m}^{k_{m}}$ is $n$-homogeneous if and only if $d_{1} k_{1}+\ldots+d_{m} k_{m}=n$. Consequently, every polynomial $f_{n}$ can be uniquely represented in the form

$$
f_{n}=\sum_{\substack{d_{1} k_{1}+\ldots+d_{m} k_{m}=n \\ k_{1}, \ldots, k_{m} \in \mathbb{Z}_{+}}} \alpha_{k_{1}, \ldots, k_{m}}^{(n)} P_{1}^{k_{1}} P_{2}^{k_{2}} \cdots P_{m}^{k_{m}},
$$

where $\alpha_{k_{1}, \ldots, k_{m}}^{(n)} \in \mathbb{C}$. Thus, every function $f \in \mathcal{A}(X)$ can be uniquely represented in the form

$$
f=\sum_{n=0}^{\infty} \sum_{\substack{d_{1} k_{1}+\ldots+d_{m} k_{m}=n \\ k_{1}, \ldots, k_{m} \in \mathbb{Z}_{+}}} \alpha_{k_{1}, \ldots, k_{m}}^{(n)} P_{1}^{k_{1}} P_{2}^{k_{2}} \cdots P_{m}^{k_{m}}
$$

where $\alpha_{k_{1}, \ldots, k_{m}}^{(n)} \in \mathbb{C}$.

Let us define a mapping $J: \mathcal{A}(X) \rightarrow H\left(\mathbb{C}^{m}\right)$ in the following way. Let $f \in \mathcal{A}(X)$. Then $f$ can be represented in the form (4). We set

$$
J(f)(z)=\sum_{n=0}^{\infty} \sum_{\substack{d_{1} k_{1}+\ldots+d_{m} k_{m}=n \\ k_{1}, \ldots, k_{m} \in \mathbb{Z}_{+}}} \alpha_{k_{1}, \ldots, k_{m}}^{(n)} z_{1}^{k_{1}} z_{2}^{k_{2}} \cdots z_{m}^{k_{m}},
$$

where $z=\left(z_{1}, \ldots, z_{m}\right) \in \mathbb{C}^{m}$. Let us show that the function $J(f)$ belongs to the Fréchet algebra $H\left(\mathbb{C}^{m}\right)$ for every $f \in \mathcal{A}(X)$. By the conditions of the theorem, there exists $C>0$ such that for every vector $z=\left(z_{1}, \ldots, z_{m}\right) \in \mathbb{C}^{m}$ there exists $x_{z} \in X$ such that $\left\|x_{z}\right\| \leq C\|z\|_{\infty}$ and $P_{j}\left(x_{z}\right)=z_{j}$ for every $j \in\{1, \ldots, m\}$. Therefore, taking into account equalities (4) and (5),

$$
J(f)(z)=f\left(x_{z}\right)
$$

for every $z \in \mathbb{C}^{m}$. Since the function $f$ is well-defined on the space $X$, by (6), the function $J(f)$ is well-defined on the space $\mathbb{C}^{m}$. Therefore the series in the right hand side of the equality (5) is convergent for every $z \in \mathbb{C}^{m}$. Consequently, the function $J(f)$ is analytic on the space $\mathbb{C}^{m}$, that is, $J(f) \in H\left(\mathbb{C}^{m}\right)$.

Let us prove that the mapping $J$ is an isomorphism between Fréchet algebras $\mathcal{A}(X)$ and $H\left(\mathbb{C}^{m}\right)$. It can be checked that the mapping $J$ is linear and multiplicative.

Let us prove that $J$ is injective. Note that for every function $f \in \mathcal{A}(X)$ and for every element $x \in X$ the following equality holds

$$
f(x)=J(f)\left(\left(P_{1}(x), P_{2}(x), \ldots, P_{m}(x)\right)\right) .
$$

Let functions $f, g \in \mathcal{A}(X)$ be different. Then there exists $x_{0} \in X$ such that $f\left(x_{0}\right) \neq g\left(x_{0}\right)$. Therefore, by (7),

$$
J(f)\left(\left(P_{1}\left(x_{0}\right), P_{2}\left(x_{0}\right), \ldots, P_{m}\left(x_{0}\right)\right)\right) \neq J(g)\left(\left(P_{1}\left(x_{0}\right), P_{2}\left(x_{0}\right), \ldots, P_{m}\left(x_{0}\right)\right)\right) .
$$

Thus, $J(f) \neq J(g)$. Hence, the mapping $J$ is injective.

Let us prove that the mapping $J$ is continuous. Let $r>0$. For $f \in \mathcal{A}(X)$ we have

$$
\|J(f)\|_{r}=\sup _{\|z\|_{\infty} \leq r}|J(f)(z)| .
$$


Taking into account the condition 3) and the equality (6), we obtain

$$
\sup _{\|z\|_{\infty} \leq r}|J(f)(z)|=\sup _{\|z\|_{\infty} \leq r}\left|f\left(x_{z}\right)\right| \leq \sup _{\|x\| \leq C r}|f(x)|=\|f\|_{C r} .
$$

Therefore, for every $r>0$ and $f \in \mathcal{A}(X)$, we have $\|J(f)\|_{r} \leq\|f\|_{C r}$. Thus, the mapping $J$ is continuous.

Let us prove that the mapping $J$ is surjective. First we prove some auxiliary fact. Let $r>0$ and $f \in \mathcal{A}(X)$. By (7),

$$
\|f\|_{r}=\sup _{\|x\| \leq r}|f(x)|=\sup _{\|x\| \leq r}\left|J(f)\left(\left(P_{1}(x), P_{2}(x), \ldots, P_{m}(x)\right)\right)\right| .
$$

By (1), $\left|P_{j}(x)\right| \leq\left\|P_{j}\right\|\|x\|^{d_{j}}$ for every $x \in X$ and $j \in\{1, \ldots, m\}$. Therefore, if $\|x\| \leq r$, then

$$
\left\|\left(P_{1}(x), P_{2}(x), \ldots, P_{m}(x)\right)\right\|_{\infty} \leq \rho(r)
$$

where

$$
\rho(r)=\max _{1 \leq j \leq m}\left(\left\|P_{j}\right\| r^{d_{j}}\right)
$$

Therefore

$$
\sup _{\|x\| \leq r}\left|J(f)\left(\left(P_{1}(x), P_{2}(x), \ldots, P_{m}(x)\right)\right)\right| \leq \sup _{\|z\|_{\infty} \leq \rho(r)}|J(f)(z)|=\|J(f)\|_{\rho(r)} .
$$

Hence, for every $r>0$ and $f \in \mathcal{A}(X)$,

$$
\|f\|_{r} \leq\|J(f)\|_{\rho(r)} .
$$

Now we prove that $J$ is surjective. Let $g \in H\left(\mathbb{C}^{m}\right)$. Let us construct a function $f \in \mathcal{A}(X)$ such that $J(f)=g$. Since the function $g$ belongs to the algebra $H\left(\mathbb{C}^{m}\right)$, it can be represented as the convergent on $\mathbb{C}^{m}$ Taylor series

$$
g(z)=\sum_{n=0}^{\infty} \sum_{\substack{l_{1}+\ldots+l_{m}=n \\ l_{1}, \ldots, l_{m} \in \mathbb{Z}_{+}}} \beta_{l_{1}, \ldots, l_{m}} z_{1}^{l_{1}} z_{2}^{l_{2}} \cdots z_{m}^{l_{m}}
$$

where $z=\left(z_{1}, \ldots, z_{m}\right) \in \mathbb{C}^{m}$ and $\beta_{l_{1}, \ldots, l_{m}} \in \mathbb{C}$. For every $N \in \mathbb{N}$ let us define a polynomial $g_{N}: \mathbb{C}^{m} \rightarrow \mathbb{C}$ by

$$
g_{N}(z)=\sum_{n=0}^{N} \sum_{\substack{l_{1}+\ldots+l_{m}=n \\ l_{1}, \ldots, l_{m} \in \mathbb{Z}_{+}}} \beta_{l_{1}, \ldots, l_{m}} z_{1}^{l_{1}} z_{2}^{l_{2}} \cdots z_{m}^{l_{m}}
$$

where $z=\left(z_{1}, \ldots, z_{m}\right) \in \mathbb{C}^{m}$. Also we define a polynomial $f_{N}: X \rightarrow \mathbb{C}$ by

$$
f_{N}(x)=\sum_{n=0}^{N} \sum_{\substack{l_{1}+\ldots+l_{m}=n \\ l_{1}, \ldots, l_{m} \in \mathbb{Z}_{+}}} \beta_{l_{1}, \ldots, l_{m}} P_{1}^{l_{1}}(x) P_{2}^{l_{2}}(x) \cdots P_{m}^{l_{m}}(x),
$$

where $x \in X$. Note that $f_{N} \in \mathcal{A}(X)$ and $J\left(f_{N}\right)=g_{N}$ for every $N \in \mathbb{N}$. Also note that the sequence $\left\{g_{N}\right\}_{N=1}^{\infty}$ converges to the function $g$ with respect to the topology of the Fréchet algebra $H\left(\mathbb{C}^{m}\right)$. By $(8)$, for every $r>0, j, N \in \mathbb{N}$,

$$
\left\|f_{N+j}-f_{N}\right\|_{r} \leq\left\|g_{N+j}-g_{N}\right\|_{\rho(r)},
$$


therefore, the fundamentality of the sequence $\left\{g_{N}\right\}_{N=1}^{\infty}$ implies the fundamentality of the sequence $\left\{f_{N}\right\}_{N=1}^{\infty}$. Since the algebra $\mathcal{A}(X)$ is complete, it follows that the sequence $\left\{f_{N}\right\}_{N=1}^{\infty}$ converges to some function $f \in \mathcal{A}(X)$. By the continuity of the mapping $J$,

$$
J(f)=\lim _{N \rightarrow \infty} J\left(f_{N}\right)=\lim _{N \rightarrow \infty} g_{N}=g .
$$

Thus, the mapping $J$ is surjective.

Hence, the mapping $J$ is linear, multiplicative, bijective and continuous. The continuity of the inverse mapping $J^{-1}$ follows from (8). Thus, the mapping $J$ is an isomorphism.

Let us describe the spectrum $\mathcal{M}(\mathcal{A}(X))$ of the Fréchet algebra $\mathcal{A}(X)$. Let us show that every character of the Fréchet algebra $\mathcal{A}(X)$ is a point-evaluation functional at some point of the space $X$. Let $\varphi \in \mathcal{M}(\mathcal{A}(X))$. Let

$$
z=\left(\varphi\left(P_{1}\right), \varphi\left(P_{2}\right), \ldots, \varphi\left(P_{m}\right)\right) .
$$

By the conditions of the theorem, there exists $x_{z} \in X$ such that $P_{j}\left(x_{z}\right)=\varphi\left(P_{j}\right)$ for every $j \in\{1, \ldots, m\}$. Consider the action of characters $\varphi$ and $\delta_{x_{z}}$ to the arbitrary function $f \in \mathcal{A}(X)$. The function $f$ can be represented in the form (4). Then

$$
\begin{aligned}
\delta_{x_{z}}(f)=\sum_{n=0}^{\infty} \sum_{\substack{d_{1} k_{1}+\ldots+d_{m} k_{m}=n \\
k_{1}, \ldots, k_{m} \in \mathbb{Z}_{+}}} \alpha_{k_{1}, \ldots, k_{m}}^{(n)}\left(P_{1}\left(x_{z}\right)\right)^{k_{1}}\left(P_{2}\left(x_{z}\right)\right)^{k_{2}} \cdots\left(P_{m}\left(x_{z}\right)\right)^{k_{m}} \\
\left.=\sum_{n=0}^{\infty} \sum_{\substack{d_{1} k_{1}+\ldots+d_{m} k_{m}=n \\
k_{1}, \ldots, k_{m} \in \mathbb{Z}_{+}}} \alpha_{k_{1}, \ldots, k_{m}}^{(n)}\left(\varphi\left(P_{1}\right)\right)^{k_{1}}\left(\varphi\left(P_{2}\right)\right)\right)^{k_{2}} \cdots\left(\varphi\left(P_{m}\right)\right)^{k_{m}} .
\end{aligned}
$$

On the other hand, by the continuity, the linearity and the multiplicativity of $\varphi$, we have

$$
\left.\varphi(f)=\sum_{n=0}^{\infty} \sum_{\substack{d_{1} k_{1}+\ldots+d_{m} k_{m}=n \\ k_{1}, \ldots, k_{m} \in \mathbb{Z}_{+}}} \alpha_{k_{1}, \ldots, k_{m}}^{(n)}\left(\varphi\left(P_{1}\right)\right)^{k_{1}}\left(\varphi\left(P_{2}\right)\right)\right)^{k_{2}} \cdots\left(\varphi\left(P_{m}\right)\right)^{k_{m}} .
$$

Thus, $\varphi(f)=\delta_{x_{z}}(f)$ for every function $f \in \mathcal{A}(X)$, that is, $\varphi=\delta_{x_{z}}$. Therefore the spectrum $\mathcal{M}(\mathcal{A}(X))$ is the set of all point-evaluation functionals at points of the space $X$. This completes the proof.

The following simple lemma will be used for proving that the subalgebra of symmetric functions is closed in the algebra $H_{b}\left(\left(L_{p}(\Omega)\right)^{n}\right)$.

Lemma 3. Let $X$ be a complex Banach space. Let a sequence of functions $\left\{f_{n}\right\}_{n=1}^{\infty} \subset H_{b}(X)$ converges to some function $f \in H_{b}(X)$. Let $A: X \rightarrow X$ be an arbitrary mapping. If $x \in X$ is such that $f_{n}(A(x))=f_{n}(x)$ for every $n \in \mathbb{N}$, then $f(A(x))=f(x)$.

Proof. Let $x \in X$ be such that $f_{n}(A(x))=f_{n}(x)$ for every $n \in \mathbb{N}$. Let $r=\max \{\|x\|,\|A(x)\|\}$. Since the sequence $\left\{f_{n}\right\}_{n=1}^{\infty}$ converges to $f$, it follows that, in particular, $\lim _{n \rightarrow \infty}\left\|f_{n}-f\right\|_{r}=0$, that is,

$$
\lim _{n \rightarrow \infty} \sup _{\|y\| \leq r}\left|f(y)-f_{n}(y)\right|=0
$$

By (9), taking into account the inequalities $\|x\| \leq r$ and $\|A(x)\| \leq r$, we obtain

$$
\lim _{n \rightarrow \infty} f_{n}(x)=f(x) \text { and } \lim _{n \rightarrow \infty} f_{n}(A(x))=f(A(x)) .
$$

Since $f_{n}(A(x))=f_{n}(x)$ for every $n \in \mathbb{N}$, it follows that $\lim _{n \rightarrow \infty} f_{n}(x)=f(A(x))$. By the uniqueness of the limit of a sequence, $f(x)=f(A(x))$. This completes the proof. 
Corollary 3. The algebra $H_{b s}\left(\left(L_{p}(\Omega)\right)^{n}\right)$ is a closed subalgebra of the Fréchet algebra $H_{b}\left(\left(L_{p}(\Omega)\right)^{n}\right)$.

Proof. By Lemma 3, if the sequence of symmetric functions $\left\{f_{n}\right\}_{n=1}^{\infty} \subset H_{b}\left(\left(L_{p}(\Omega)\right)^{n}\right)$ converges to $f \in H_{b}\left(\left(L_{p}(\Omega)\right)^{n}\right)$, then $f$ is symmetric. Thus, $H_{b s}\left(\left(L_{p}(\Omega)\right)^{n}\right)$ is closed in $H_{b}\left(\left(L_{p}(\Omega)\right)^{n}\right)$.

Lemma 4. Let $M$ be a nonempty subset of the set $\left\{k \in \mathbb{Z}_{+}^{n}: 1 \leq|k| \leq p\right\}$. There exists a constant $C>0$ such that for every $z=\left(z_{k}\right)_{k \in M} \in \mathbb{C}^{M}$ there exists an element $x_{z} \in\left(L_{p}[0,1]\right)^{n}$ such that $\left\|x_{z}\right\|_{p, n,[0,1]} \leq C\|z\|_{\infty}$ and $R_{k,[0,1]}\left(x_{z}\right)=z_{k}$ for every $k \in M$.

Proof. By Theorem 3, for the set $M$ there exist numbers $l \in \mathbb{N}$ and $\rho_{M}>0$ such that for every $\xi=\left(\xi_{k}\right)_{k \in M} \in \mathbb{C}^{M}$, for which $\|\xi\|_{\infty}<1$, there exists a sequence $y_{\xi} \in c_{00}^{\left(2^{l}\right)}\left(\mathbb{C}^{n}\right)$ such that $\left\|y_{\xi}\right\|_{\ell_{p}}<\rho_{M}$ and $H_{k}^{\left(2^{l}\right)}\left(y_{\xi}\right)=\xi_{k}$ for every $k \in M$, where polynomials $H_{k}^{\left(2^{l}\right)}$ are defined by (2).

Let $z=\left(z_{k}\right)_{k \in M}$ be an arbitrary element of $\mathbb{C}^{M}$. Consider the case $\|z\|_{\infty}>0$. Let

$$
a=2^{l+1}\|z\|_{\infty} \quad \text { and } \quad \xi=\left(\frac{2^{l} z_{k}}{a^{|k|}}\right)_{k \in M} .
$$

Then

$$
\|\xi\|_{\infty}=\max _{k \in M}\left|\frac{2^{l} z_{k}}{a^{|k|}}\right| \leq \max _{k \in M}\left|\frac{2^{l} z_{k}}{a}\right|=\frac{2^{l}\|z\|_{\infty}}{a}=\frac{1}{2}<1 .
$$

Therefore there exists a sequence $y_{\xi} \in c_{00}^{\left(2^{l}\right)}\left(\mathbb{C}^{n}\right)$ such that $\left\|y_{\xi}\right\|_{\ell_{p}}<\rho_{M}$ and

$$
H_{k}^{\left(2^{l}\right)}\left(y_{\xi}\right)=\frac{2^{l} z_{k}}{a^{|k|}}
$$

for every $k \in M$. Let

$$
x_{z}=J_{2^{l}}\left(a y_{\xi}\right),
$$

where the mapping $J_{2^{l}}$ is defined by the equality (3). By Lemma 1, taking into account the inequality $\left\|y_{\xi}\right\|_{\ell_{p}}<\rho_{M}$, we have

$$
\left\|x_{z}\right\|_{p, n,[0,1]}=\frac{1}{2^{l / p}}\left\|a y_{\xi}\right\|_{\ell_{p}}<\frac{a \rho_{M}}{2^{l / p}}=\frac{2^{l+1} \rho_{M}}{2^{l / p}}\|z\|_{\infty}
$$

Therefore

$$
\left\|x_{z}\right\|_{p, n,[0,1]} \leq C\|z\|_{\infty}
$$

where $C=\frac{2^{l+1} \rho_{M}}{2^{l / p}}$. Note that the constant $C$ does not depend on $z$. By Lemma 2, taking into account the equality (10) and the $|k|$-homogeneity of the polynomial $H_{k}^{\left(2^{l}\right)}$, we obtain

$$
R_{k,[0,1]}\left(x_{z}\right)=\frac{1}{2^{l}} H_{k}^{\left(2^{l}\right)}\left(a y_{\xi}\right)=\frac{1}{2^{l}} a^{|k|} H_{k}^{\left(2^{l}\right)}\left(y_{\xi}\right)=\frac{1}{2^{l}} a^{|k|} \frac{2^{l} z_{k}}{a^{|k|}}=z_{k}
$$

for every $k \in M$. Consider the case $\|z\|_{\infty}=0$. In this case we set $x_{z} \equiv 0$. Evidently, $R_{k,[0,1]}\left(x_{z}\right)=$ 0 for every $k \in M$. This completes the proof. 
Lemma 5. Let $p \in \mathbb{N}$. Let $M=\left\{k \in \mathbb{Z}_{+}^{n}:|k|=p\right\}$. There exists a constant $C>0$ such that for every $z=\left(z_{k}\right)_{k \in M} \in \mathbb{C}^{M}$ there exists an element $\tilde{x}_{z} \in\left(L_{p}[0,+\infty)\right)^{n}$ such that $\left\|\tilde{x}_{z}\right\|_{p, n,[0,+\infty)} \leq$ $C\|z\|_{\infty}$ and $R_{k,[0,+\infty)}\left(\tilde{x}_{z}\right)=z_{k}$ for every $k \in M$.

Proof. Let us define a mapping $I:\left(L_{p}[0,1]\right)^{n} \rightarrow\left(L_{p}[0,+\infty)\right)^{n}$ by

$$
I(x)(t)= \begin{cases}x(t), & \text { if } t \in[0,1] \\ (0, \ldots, 0), & \text { if } t \in(1,+\infty),\end{cases}
$$

where $x \in\left(L_{p}[0,1]\right)^{n}, t \in[0,1]$. It can be checked that

$$
\|I(x)\|_{p, n,[0,+\infty)}=\|x\|_{p, n,[0,1]}
$$

for every $x \in\left(L_{p}[0,1]\right)^{n}$ and

$$
R_{k,[0,+\infty)}(I(x))=R_{k,[0,1]}(x)
$$

for every $x \in\left(L_{p}[0,1]\right)^{n}, k \in \mathbb{Z}_{+}^{n}$ such that $|k|=p$.

By Lemma 4 , there exists a constant $C>0$ such that for every $z=\left(z_{k}\right)_{k \in M} \in \mathbb{C}^{M}$ there exists an element $x_{z} \in\left(L_{p}[0,1]\right)^{n}$ such that $\left\|x_{z}\right\|_{p, n,[0,1]} \leq C\|z\|_{\infty}$ and $R_{k,[0,1]}\left(x_{z}\right)=z_{k}$ for every $k \in M$. For $z=\left(z_{k}\right)_{k \in M} \in \mathbb{C}^{M}$, let $\tilde{x}_{z}=I\left(x_{z}\right)$. Then

$$
\left\|\tilde{x}_{z}\right\|_{p, n,[0,+\infty)}=\left\|x_{z}\right\|_{p, n,[0,1]} \leq C\|z\|_{\infty}
$$

and

$$
R_{k,[0,+\infty)}\left(\tilde{x}_{z}\right)=R_{k,[0,1]}\left(x_{z}\right)=z_{k}
$$

for every $k \in M$. This completes the proof.

Integral Cauchy formula [11, Corollary 7.3, p. 47] implies the following corollary.

Corollary 4. Let $f \in H_{b s}\left(\left(L_{p}(\Omega)\right)^{n}\right)$. Then every term of the Taylor series of $f$ is a symmetric continuous homogeneous polynomial.

Theorem 1 and Corollary 4 imply the following corollary.

Corollary 5. Let $f \in H_{b s}\left(\left(L_{p}[0,1]\right)^{n}\right)$. Then every term of the Taylor series of $f$ can be uniquely represented as an algebraic combination of polynomials $R_{k,[0,1]}$, where $k \in \mathbb{Z}_{+}^{n}$ such that $1 \leq|k| \leq p$.

Analogically, Theorem 2 and Corollary 4 imply the following corollary.

Corollary 6. Let $p \in \mathbb{N}$. Let $f \in H_{b s}\left(\left(L_{p}[0,+\infty)\right)^{n}\right)$. Then every term of the Taylor series of $f$ can be uniquely represented as an algebraic combination of polynomials $R_{k,[0,+\infty)}$, where $k \in \mathbb{Z}_{+}^{n}$ such that $|k|=p$.

Theorem 5. The Fréchet algebra $H_{b s}\left(\left(L_{p}[0,1]\right)^{n}\right)$ is isomorphic to the Fréchet algebra $H\left(\mathbb{C}^{m}\right)$, where $m$ is the cardinality of the set of multi-indexes

$$
M=\left\{k \in \mathbb{Z}_{+}^{n}: 1 \leq|k| \leq p\right\} .
$$

The spectrum of the Fréchet algebra $H_{b s}\left(\left(L_{p}[0,1]\right)^{n}\right)$ coincides with the set of all point-evaluation functionals at points of the space $\left(L_{p}[0,1]\right)^{n}$. 
Proof. Let $X=\left(L_{p}[0,1]\right)^{n}, \mathcal{A}(X)=H_{b s}\left(\left(L_{p}[0,1]\right)^{n}\right)$ and $\Gamma=\left\{R_{k,[0,1]}: k \in M\right\}$. Let us check the conditions of Theorem 4 . Since $R_{k,[0,1]}$ is a continuous $|k|$-homogeneous polynomial for every $k \in M$, it follows that condition 1) is satisfied. By Corollary 1, condition 2) is satisfied. By Lemma 4, condition 3$)$ is satisfied. By Corollary $3, H_{b s}\left(\left(L_{p}[0,1]\right)^{n}\right)$ is a closed subalgebra of $H_{b}\left(\left(L_{p}[0,1]\right)^{n}\right)$. By Corollary 5 , every term of the Taylor series of every $f \in H_{b s}\left(\left(L_{p}[0,1]\right)^{n}\right)$ can be uniquely represented as an algebraic combination of polynomials $R_{k,[0,1]}$, where $k \in$ $M$. Therefore, by Theorem 4 , the Fréchet algebra $H_{b s}\left(\left(L_{p}[0,1]\right)^{n}\right)$ is isomorphic to the Fréchet algebra $H\left(\mathbb{C}^{m}\right)$, where $m$ is the cardinality of the set $M$ and the spectrum of the Fréchet algebra $H_{b s}\left(\left(L_{p}[0,1]\right)^{n}\right)$ coincides with the set of all point-evaluation functionals at points of the space $\left(L_{p}[0,1]\right)^{n}$. This completes the proof.

Theorem 6. Let $p \in \mathbb{N}$. The Fréchet algebra $H_{b s}\left(\left(L_{p}[0,+\infty)^{n}\right)\right.$ is isomorphic to the Fréchet algebra $H\left(\mathbb{C}^{m}\right)$, where $m$ is the cardinality of the set of multi-indexes

$$
M=\left\{k \in \mathbb{Z}_{+}^{n}:|k|=p\right\} .
$$

The spectrum of the Fréchet algebra $H_{b s}\left(\left(L_{p}[0,+\infty)^{n}\right)\right.$ coincides with the set of all pointevaluation functionals at points of the space $\left(L_{p}[0,+\infty)\right)^{n}$.

Proof. Let $X=\left(L_{p}[0,+\infty)\right)^{n}, \mathcal{A}(X)=H_{b s}\left(\left(L_{p}[0,+\infty)\right)^{n}\right)$ and $\Gamma=\left\{R_{k,[0,+\infty)}: k \in M\right\}$. Let us check the conditions of Theorem 4 . Since $R_{k,[0,+\infty)}$ is a continuous $|k|$-homogeneous polynomial for every $k \in M$, it follows that condition 1) is satisfied. By Corollary 2, condition 2) is satisfied. By Lemma 4, condition 3) is satisfied. By Corollary 3, $H_{b s}\left(\left(L_{p}[0,+\infty)\right)^{n}\right)$ is a closed subalgebra of $H_{b}\left(\left(L_{p}[0,+\infty)\right)^{n}\right)$. By Corollary 6, every term of the Taylor series of every $f \in H_{b s}\left(\left(L_{p}[0,+\infty)\right)^{n}\right)$ can be uniquely represented as an algebraic combination of polynomials $R_{k,[0,+\infty)}$, where $k \in M$. Therefore, by Theorem 4 , the Fréchet algebra $H_{b s}\left(\left(L_{p}[0,+\infty)\right)^{n}\right)$ is isomorphic to the Fréchet algebra $H\left(\mathbb{C}^{m}\right)$, where $m$ is the cardinality of the set $M$ and the spectrum of the Fréchet algebra $H_{b s}\left(\left(L_{p}[0,+\infty)\right)^{n}\right)$ coincides with the set of all point-evaluation functionals at points of the space $\left(L_{p}[0,+\infty)\right)^{n}$. This completes the proof.

\section{References}

[1] Alencar R., Aron R., Galindo P., Zagorodnyuk A. Algebras of symmetric holomorphic functions on $\ell_{p}$. Bull. Lond. Math. Soc. 2003, 35 (2), 55-64. doi:10.1112/S0024609302001431

[2] Aron R., Galindo P., Pinasco D., Zalduendo I. Group-symmetric holomorphic functions on a Banach space. Bull. Lond. Math. Soc. 2016, 48 (5), 779-796. doi:10.1112/blms/bdw043

[3] Chernega I., Galindo P., Zagorodnyuk A. Some algebras of symmetric analytic functions and their spectra. Proc. Edinb. Math. Soc. 2012, 55 (1), 125-142. doi:10.1017/S0013091509001655

[4] Chernega I., Holubchak O., Novosad Z., Zagorodnyuk A. Continuity and hypercyclicity of composition operators on algebras of symmetric analytic functions on Banach spaces. Eur. J. Math. 2020, 6 (1), $153-163$. doi:10.1007/s40879-019-00390-z

[5] Galindo P., Vasylyshyn T., Zagorodnyuk A. The algebra of symmetric analytic functions on $L_{\infty}$. Proc. Roy. Soc. Edinburgh Sect. A 2017, 147 (4), 743-761. doi:10.1017/S0308210516000287

[6] Galindo P., Vasylyshyn T., Zagorodnyuk A. Symmetric and finitely symmetric polynomials on the spaces $\ell_{\infty}$ and $L_{\infty}[0,+\infty)$. Math. Nachr. 2018, 291 (11-12), 1712-1726. doi:10.1002/mana.201700314

[7] Galindo P., Vasylyshyn T., Zagorodnyuk A. Analytic structure on the spectrum of the algebra of symmetric analytic functions on $L_{\infty}$. Rev. R. Acad. Cienc. Exactas Fís. Nat. Ser. A Mat. 2020, 114, Article number 56. doi:10.1007/s13398-020-00791-w 
[8] González M., Gonzalo R., Jaramillo J. A. Symmetric polynomials on rearrangement invariant function spaces. J. Lond. Math. Soc. 1999, 59 (2), 681-697. doi:10.1112/S0024610799007164

[9] Kravtsiv V., Vasylyshyn T., Zagorodnyuk A. On algebraic basis of the algebra of symmetric polynomials on $\ell_{p}\left(\mathbb{C}^{n}\right)$. J. Funct. Spaces 2017, 2017, Article ID 4947925, 8 p. doi:10.1155/2017/4947925

[10] Nemirovskii A. S., Semenov S. M. On polynomial approximation of functions on Hilbert space. Mat. USSR Sb. 1973, 21 (2), 255-277. doi:10.1070/SM1973v021n02ABEH002016

[11] Mujica J. Complex Analysis in Banach Spaces. North Holland, 1986.

[12] Vasylyshyn T. Algebras of entire symmetric functions on spaces of Lebesgue measurable essentially bounded functions. J. Math. Sci. (N.Y.) 2020, 246 (2), 264-276. doi:10.1007/s10958-020-04736-x

[13] Vasylyshyn T. Symmetric functions on spaces $\ell_{p}\left(\mathbb{R}^{n}\right)$ and $\ell_{p}\left(\mathbb{C}^{n}\right)$. Carpathian Math. Publ. 2020, 12 (1), 5-16. doi:10.15330/cmp.12.1.5-16

[14] Vasylyshyn T. Symmetric polynomials on $\left(L_{p}\right)^{n}$. Eur. J. Math. 2020, 6 (1), 164-178. doi:10.1007/s40879-018-0268-3

[15] Vasylyshyn T. V. Symmetric polynomials on the Cartesian power of $L_{p}$ on the semi-axis. Mat. Stud. 2018, 50 (1), 93-104. doi:10.15330/ms.50.1.93-104

[16] Vasylyshyn T. V. The algebra of symmetric polynomials on $\left(L_{\infty}\right)^{n}$. Mat. Stud. 2019, 52 (1), 71-85. doi: $10.30970 / \mathrm{ms} .52 .1 .71-85$

[17] Vasylyshyn T. V., Strutinskii M. M. Algebras of symmetric *-polynomials in the space $\mathbb{C}^{2}$. J. Math. Sci. (N.Y.) 2021, 253 (1), 40-53. doi:10.1007/s10958-021-05211-x

[18] Vasylyshyn T. V., Zagorodnyuk A. V. Symmetric polynomials on the Cartesian power of the real Banach space $L_{\infty}[0,1]$. Mat. Stud. 2020, 53 (2), 192-205. doi:10.30970/ms.53.2.192-205

[19] Vasylyshyn T., Zagorodnyuk A. Continuous symmetric 3-homogeneous polynomials on spaces of Lebesgue measurable essentially bounded functions. Methods Funct. Anal. Topology 2018, 24 (4), 381-398.

Received 04.02.2021

Василишин Т.В. Алгебри симетричних аналітичних функиій на декартових степенях інтегровних за Аебегом у степені $p \in[1,+\infty)$ функиій // Карпатські матем. публ. - 2021. — Т.13, №2. - C. 340-351.

Роботу присвячено дослідженню алгебр Фреше симетричних (інваріантних щодо дії композиції кожної координати аргументу із довільною бієкцією області визначення координат, яка зберігає міру) аналітичних функцій на декартових степенях комплексних банахових просторів інтегровних за Аебегом у степені $p \in[1,+\infty)$ комплекснозначних функцій на відрізку $[0,1]$ і на півосі. Показано, що алгебра Фреше всіх симетричних аналітичних цілих комплекснозначних функцій обмеженого типу на $n$-тому декартовому степені комплексного банахового простору $L_{p}[0,1]$ всіх інтегровних за чних функцій на вілрізку $[0,1] €$ ізоморфною до алгебри Фреше всіх аналітичних цілих функцій на просторі $\mathbb{C}^{m}$, де $m$ - це потужність алгебраїчного базису алгебри всіх симетричних неперервних комплекснозначних поліномів на цьому декартовому степені. Аналогічний результат доведено для алгебри Фреше всіх симетричних аналітичних цілих комплекснозначних функцій обмеженого типу на $n$-тому декартовому степені комплексного банахового простору $L_{p}[0,+\infty)$ всіх інтегровних за $\Lambda$ ебегом у степені $p \in[1,+\infty)$ комплекснозначних функцій на півосі $[0,+\infty)$.

Ключові слова і фрази: симетричний поліном, симетрична аналітична функція, алгебра Фреше аналітичних функцій. 